\title{
Marie DELAPLACE et Maria GRAVARI-BARBAS (dir.), Nouveaux territoires touristiques. Invention, reconfigurations, repositionnement
}

Coll. « Tourisme », Presses de l'université du Québec, 2017, 252 pages

Philippe Duhamel

\section{OpenEdition}

\section{Journals}

Édition électronique

URL : http://journals.openedition.org/tourisme/1415

DOI : 10.4000/tourisme.1415

ISSN : 2492-7503

\section{Éditeur}

Éditions touristiques européennes

\section{Référence électronique}

Philippe Duhamel, « Marie delaplace et Maria gravari-Barbas (dir.), Nouveaux territoires touristiques. Invention, reconfigurations, repositionnement », Mondes du Tourisme [En ligne], 13 | 2017, mis en ligne le 30 décembre 2017, consulté le 23 septembre 2020. URL : http://journals.openedition.org/tourisme/ 1415 ; DOI : https://doi.org/10.4000/tourisme.1415

Ce document a été généré automatiquement le 23 septembre 2020.

Mondes du tourisme est mis à disposition selon les termes de la licence Creative Commons Attribution - Pas d'Utilisation Commerciale - Pas de Modification 4.0 International. 


\section{Marie DELAPLACE et Maria GRAVARI- BARBAS (dir.), Nouveaux territoires touristiques. Invention, reconfigurations, repositionnement}

Coll. « Tourisme », Presses de l'université du Québec, 2017, 252 pages

Philippe Duhamel

\section{RÉFÉRENCE}

Marie DELAPLACE et Maria GRAVARI-BARBAS (dir.), Nouveaux territoires touristiques. Invention, reconfigurations, repositionnement, Coll. « Tourisme », Presses de l'université du Québec, 2017, 252 pages

L'ouvrage dirigé par Marie Delaplace et Maria Gravari-Barbas aborde un sujet d'importance aujourd'hui, celui du renouvellement de l'offre dans un contexte de développement touristique intense $d u$ fait à la fois de la mise en tourisme de nouveaux territoires et de la densification d'autres, anciennement «touristifiés ». Pour aborder cette question, les auteures ont privilégié une approche en trois axes de réflexion, qui constitue le plan de l'ouvrage. L'ouvrage montre tout d'abord « combien le tourisme a contribué et contribue à 'défricher' certains territoires»; il décline ensuite « les changements affectant les valorisations touristiques des territoires ruraux, littoraux et de montagnes »; il présente enfin le « renouveau du tourisme en ville ». Au total, ce sont neuf textes qui sont proposés avec des auteur-e-s d'horizon franco-québécois, géographes, sociologues, économistes, urbanistes ou ingénieurs - ce qui permet des approches diversifiées et intéressantes le plus souvent.

2 L'ensemble présente un réel intérêt par la dimension exemplaire des textes retenus, qui permettent souvent d'alimenter la réflexion sur les sujets qu'ils abordent. On pensera tout particulièrement au texte intéressant d'Antoine Delmas sur la mise en tourisme 
des zones polaires et le «cryotropisme » (p. 19). Même si le mot n'est pas totalement pertinent selon nous, le cas développé est réellement stimulant quant à la capacité des sociétés à donner «un sens touristique " à toutes les parties du Monde. Il en est de même pour l'approche de Jacinthe Bessière, qui vient bousculer les idées reçues autour de la recomposition des patrimoines alimentaires locaux. Elle montre combien l'ancrage dans l'histoire n'est pas forcément synonyme de passéisme et qu'il permet au contraire de produire de la «création et de l'innovation » et de "valoriser le lien à la modernité » (p. 141). Tout cela s'inscrit dans «des processus peu linéaires » qui montrent la diversité des manières de faire (p. 144).

3 Comme souvent dans les ouvrages sur le tourisme, d'autres dynamiques sont associées et relèvent plus des loisirs ou de la qualité des lieux que du tourisme lui-même. Cette impression semble particulièrement forte lorsqu'on aborde la troisième partie de l'ouvrage. Le texte de Driss Boumegoutti et Sylvie Christofle pose clairement la question de la "porosité entre tourisme et loisirs» (p.196). On aurait pu ajouter ici le texte collectif sur la « Réserve internationale de ciel étoilé du Pic du Midi » où se joue une autre porosité, celle entre "tourisme et science» (p. 63). L'organisation de l'ouvrage nuit malheureusement à l'appréciation pleine et entière de ce thème de la porosité, pourtant fondamentale aujourd'hui. On se prive d'une lecture scientifique fine et riche de l'évolution des pratiques et des phénomènes sociaux. Quant au texte de Belinda Redonda sur le tram comme objet touristique ou outil de touristification, il est le plus éloigné du titre de l'ouvrage. En effet, même si la réflexion proposée est digne d'intérêt, elle s'ajuste mal à la problématique selon nous. Ici le tram semble plus une question pour les populations et l'urbanisme des villes concernées que pour les touristes, pour l'instant. Le lien au tourisme pourrait/pourra se faire, mais ne semble pas encore effectif.

Il est dommage que certains ouvrages sur le tourisme ne parviennent pas encore à montrer les limites du tourisme. Et le fait d'intégrer sans le dire des thématiques en lisière du tourisme, ou clairement non touristiques, nourrit encore le sentiment que tout est tourisme ou qu'il n'est pas possible de sérier le phénomène touristique. Au rang des déceptions aussi, cette assertion de l'introduction dans laquelle les directrices de l'ouvrage, évoquant les textes de la première partie, affirment que le tourisme serait un « vecteur de dénaturation, voire de possible destruction des territoires qu'il investit, et contient ainsi en son germe la disparition de ce qui fait leur essence touristique». Outre le fait que les textes en question n'alimentent pas vraiment cette idée, elle nous interroge sur le choix même de ces chercheures à publier de tels textes. À quel besoin cela répond-il et qu'est-ce cela apporte aux débats scientifiques ? Rien, on le sait depuis longtemps maintenant. Alors, faut-il y voir comme Laurent Queige, l'expression de la tourismophobie ambiante alimentée aussi par les « beaux esprits » :

«Certains universitaires, ethnologues ou autres sociologues n'ont pas de mots assez acides pour accabler le tourisme de tous les maux. Ils surfent sur une vieille rengaine: regrettant l'époque bénie des 'voyageurs cultivés' du XIX ${ }^{\mathrm{e}}$ siècle, ils tirent sur le touriste contemporain, qu'il faudrait d'urgence 'réprimer ou éduquer'. L'éternel 'c'était mieux avant', version bac +6 . On s'empresse de donner des leçons à la terre entière, sans faire l'effort de s'attaquer aux racines du mal » (L'Écho touristique du 22 septembre 2017). 